\title{
Knockdown of ALDH1A3 reduces breast cancer stem cell marker CD44 via the miR-7-TGFBR2-Smad3-CD44 regulatory axis
}

\author{
MENG PAN ${ }^{1,2^{*}},{\text { MIAO } \mathrm{LI}^{2 *}, \text { MEI GUO }}^{2^{*}}$, HUIYING ZHOU ${ }^{1 *}$, HUI XU ${ }^{2}$, \\ FENGSHU ZHAO ${ }^{2}$, FENG MEI ${ }^{2}$, RUI XUE $^{2}$ and JUN DOU ${ }^{2}$ \\ ${ }^{1}$ Department of Judicial Identification, Jiangsu Province Hospital, The First Affiliated Hospital of \\ Nanjing Medical University, Nanjing, Jiangsu 210029; ${ }^{2}$ Department of Pathogenic Biology and \\ Immunology, School of Medicine, Southeast University, Nanjing, Jiangsu 210009, P.R. China
}

Received July 28, 2020; Accepted March 22, 2021

DOI: $10.3892 / \mathrm{etm} .2021 .10527$

\begin{abstract}
Inhibition of aldehyde dehydrogenase 1 family member A3 (ALDH1A3) has been revealed to lead to significant increase of microRNA (miR)-7 expression and decrease of CD44 expression in breast cancer stem cells (BCSCs), however the mechanism is not clear. The aim of the present study was to investigate the regulatory relationship between ALDH1A3, miR-7, and CD44 in BCSCs. The expression of ALDH1A3 was inhibited by small interfering RNA (siRNA or si), and the expression of miR-7 was detected by reverse transcription-quantitative polymerase chain reaction (RT-qPCR). Then, the ratio of $\mathrm{CD} 44^{+}$cells was analyzed by flow cytometry in MDA-MB-231 cells. The dual-luciferase reporter system was used to demonstrate that miR-7 binds to transforming growth factor- $\beta$ receptor 2 (TGFBR2) 3'UTR, and ChIP-PCR determined whether the transcription factor Smad3 binds to the upstream regulatory region of the CD44 promoter. The results revealed that siALDH1A3 downregulated ALDH1A3 and promoted miR-7 expression, which resulted in downregulation of CD44 expression. siALDH1A3 also downregulated the CD44 expression on the surface of MDA-MB-231 cells and inhibited the G2/M phase in BCSCs as analyzed by flow cytometry. In addition, lenti-miR-7 cells transfected with TGF- $\beta 1+$ SB 431542 revealed that lenti-miR-7 inhibited the TGF- $\beta 1$ pathway by inhibiting Smad2/3/4 expression and, thus, downregulated CD44 expression. miR-7 was revealed to directly bind to the TGFBR2 3'UTR through dual-luciferase reporter assay, and $\mathrm{Smad} 3$, a transcription factor, through ChIP-PCR was demonstrated to bind to the upstream region
\end{abstract}

Correspondence to: Dr Jun Dou, Department of Pathogenic Biology and Immunology, School of Medicine, Southeast University, 87 Ding Jiaqiao Road, Nanjing, Jiangsu 210009, P.R. Chin

E-mail: njdoujun@seu.edu.cn

${ }^{*}$ Contributed equally

Key words: microRNA-7, breast cancer, cancer stem cells, aldehyde dehydrogenase 1 family member A3, CD44 of the CD44 promoter. These results demonstrated the existence of the ALDH1A3-miR-7-TGFBR2-Smad3-CD44 axis in MDA-MB-231 cells. RT-qPCR results of 12 breast cancer surgical specimens and SK-BR-3, MCF-7, and LD cell lines further confirmed the presence of the regulatory axis. In conclusion the findings from the present study demonstrated that the ALDH1A3-miR-7-TGFBR2-Smad3-CD44 regulatory axis was highly efficient in the inhibition of CD44 expression in BCSCs, and that the regulatory expression of ALDH1A3 and miR-7 may provide a strategy in the therapy of breast cancer.

\section{Introduction}

Breast cancer is the most common malignant disease that mainly affects females. Recurrence and metastasis result in unfavorable prognoses for breast cancer patients; up to $30 \%$ of patients succumb to this disease due to relapse and metastasis after having standard-of-care therapy (1). Therefore, novel therapies are urgently required. Cancer metastases can be attributed to multiple factors such as cancer cell biological processes that underlie the dissemination and metastatic outgrowth of cancer cells, cancer stem cells (CSCs) (2,3). MicroRNAs (miRs) are a class of small noncoding RNAs (19-22 nt) that are involved in biological processes such as proliferation, differentiation, apoptosis, and development $(4,5)$. miR-based therapeutic strategies are promising for cancer therapy. MiR-7 is an intronic microRNA that resides in the first intron of the heterogeneous ribonuclear protein $\mathrm{K}$ gene on chromosome 9 and is downregulated in different cancer types (6). Our previous study had revealed that miR-7, which was downregulated in breast CSCs (BCSCs; EpCAM ${ }^{+} \mathrm{CD} 44^{+} \mathrm{CD} 24^{-/ \mathrm{low}}$ ) isolated from the human MCF-7 and MDA-MB-231 cell lines, inhibited cell invasion and metastasis, decreased the BCSC population, and partially reversed EMT in MDA-MB-231 cells by directly targeting the oncogene, SETDB1 (7). However, the molecular mechanism by which miR-7 plays a role in BCSC subset downregulation is not clear. Due to the existence of a BCSC subset, chemotherapy, radiotherapy sensitivity, and therapeutic effects have been revealed to be decreased. It is known that the quantity of the BCSC subset is closely related to the survival of breast cancer patients (8). Therefore, it is of great significance to 
elucidate the molecular mechanism of miR-7 to reduce the amount of the BCSC subset and to use miR-7 to target BCSC in the treatment of breast cancer.

Although our latest work investigated the relationship between miR-7 and ALDH1A3, a few questions remain unanswered (9). For example, it is not known how miR-7 downregulates CD44 and what role ALDH1A3 plays in impacting CD44 expression in MDA-MB-231 cells, which leads to a decrease of the BCSC subset. In the present study, the mechanisms involved in small interfering (si)ALDH1A3 downregulation of CD44 via the TGF- $\beta 1$ pathway were explored.

\section{Materials and methods}

Cell culture and reagents. Human breast cancer cell lines MDA-MB-231, SK-BR-3, and MCF-7 were obtained from the Shanghai Institute of Biochemistry and Cell Biology, Chinese Academy of Sciences. The LD cell line was established by our laboratory (Department of Pathogenic Biology and Immunology, School of Medicine, Southeast University, Nanjing, China) from a human breast cancer postsurgery sample (8). All cells were cultured in Dulbecco's modified Eagle's medium containing 10\% fetal bovine serum (GibcoTM; Thermo Fisher Scientific, Inc.). Lipofectamine ${ }^{\mathrm{TM}} 2000$ reagent was obtained from Invitrogen; Thermo Fisher Scientific, Inc. TGF- $\beta 1$ (Novoprotein Scientific, Inc.) and SB431542 (selective inhibitor of TGF- $\beta$ RI) (cat. no. A8249; APeXBIO Technology LLC) were used to treat cells.

Human breast cancer samples. The data of 12 clinical human breast cancer postsurgery samples, were recorded in our previous study (8), and were obtained from the Department of General Surgery of Zhongda Hospital at Southeast University (Nanjing, China). The investigation was approved by the Ethics Committee at Southeast University School of Medicine, and informed consent for the use of the postsurgery samples was obtained from the donors who were breast cancer patients.

Magnetic cell sorting (MACS) for BCSCs. CD44 (cat. no. 130-095-194)/CD24 (cat. no. 130-095-951)/CD326 (cat. no. 130-061-101) antibodies conjugated to magnetic microbeads (Miltenyi Biotec $\mathrm{GmbH}$ ) were used to obtain the BCSCs from MDA-MB-231 cell lines, respectively. The isolation process was according to the manufacturer's instructions.

siRNA design and plasmid transfection. siALDH1A3 was designed based on the ALDH1A3 DNA sequence (GenBank no. NM_001128128.2) using the siDESIGN design software (http://www.dharmacon.com/). siALDH1A3 (5'-GUUCAAAAGUAUCGAAGAA-3') and siRNA-NC (5'-GGCUCUAGAAAAGCCUAUGCdTdT-3') sequences were synthesized by Guangzhou RiboBio Co., Ltd. MDA-MB-231 cells were transiently transfected using Lipofectamine ${ }^{\circledR} 2000$ transfection reagent (Invitrogen; Thermo Fisher Scientific, Inc.). A total of $1 \mu \mathrm{l}$ Lipofectamine was added to $50 \mu 1$ serum-free and antibiotic-free DMEM medium, mixed with $50 \mathrm{nM}$ siRNA at room temperature for $20 \mathrm{~min}$. The mixture was added to $1 \times 10^{5}$ cells in 24 well-plate, which were cultured at $37^{\circ} \mathrm{C}$ with $5 \% \mathrm{CO}_{2}$ for $4-6 \mathrm{~h}$ for transfection. The subsequent experiments were performed $48 \mathrm{~h}$ after transfection.

Flow cytometry (FCM). CD44-APC antibodies (1:100 dilution; cat. no. 17-0441-82; eBioscience; Thermo Fisher Scientific, Inc.) diluted in PBS were used to label $1 \times 10^{6}$ cells at $4^{\circ} \mathrm{C}$ for $30 \mathrm{~min}$ and the stained cells were analyzed using BD FACSAria (BD Biosciences) according to the manufacturer's instructions. FlowJo v10 (FlowJo LLC) was used for analysis.

Reverse transcription-quantitative polymerase chain reaction $(R T-q P C R)$. To evaluate the expression of miR-7, Smad2, Smad3, Smad4, transforming growth factor- $\beta$ receptor 2 (TGFBR2), and CD44 respectively, total cellular RNA was isolated from each sample using TRIzol ${ }^{\circledR}$ (Invitrogen; Thermo Fisher Scientific, Inc.) and used for the reverse transcription reactions (PrimeScript ${ }^{\mathrm{TM}}$ RT Master Mix; cat. no. RR036A; Takara Bio, Inc.), followed by qPCR (One Step TB Green ${ }^{\circledR}$ PrimeScript $^{\mathrm{TM}}$ RT-PCR kit; cat. no. RR066A; Takara Bio, Inc.) was performed on a StepOnePlus ${ }^{\mathrm{TM}}$ System (AB Applied Biosystems; Thermo Fisher Scientific, Inc.). The $2^{-\Delta \Delta C q}$ method was used for quantification (10). GAPDH was used as an internal control. The following primer sequences were used: miR-7 (forward, 5'-ACACTCCAGCTGGGTGGAAGACTA GTGATTT-3'; reverse, 5'-CTCAACTGGTGTCGTGGAG TCGGCAATTCAGTTGAGACAACAAA-3'), Smad2 (forward, 5'-GTCGTCCATCTTGCCATTCAC-3'; reverse, 5'-TTCCTGCCCATTCTGCTCTC-3'), Smad3 (forward, 5'-GTCGTCCATCCTGCCTTTCA-3'; reverse, 5'-GTTTCT TGACCAGGCTCTTGACC-3'), Smad4 (forward, 5'-GCT GCTGGAATTGGTGTTGATG-3'; reverse, 5'-AGGTGT TTCTTTGATGCTCTGTCT-3'), TGFBR2 (forward, 5'-GCA CGTTCAGAAGTCGGATG-3'; reverse, 5'-CTGCACCGT TGTTGTCAGTG-3'), CD44 (forward, 5'-GCCCAATGCCTT TGATGGAC-3'; reverse, 5'-CCCATGTGAGTGTCTGGT AGC-3'); GAPDH (forward, 5'-AGGTCGGTGTGAACG GATTTG-3'; reverse, 5'-GGGGTCGTTGATGGCAACA-3'); and U6 (forward, 5'-GCTTCGGCAGCACATATACTAA AAT-3'; reverse, 5'-CGCTTCACGAATTTGCGTGTCAT-3'). U6 was used as an internal control for miR-7 and GAPDH was used as an internal control for mRNA (Smad2, Smad3, Smad4, TGFBR2 and CD44) quantification.

Dual-luciferase reporter assay. Targeted binding sites of miR-7 and TGFBR2 3'UTR were predicted through TargetScan website (http://www.targetscan.org/vert_72/) (11). The full-length TGFBR2 3'UTR was amplified via PCR using PrimeSTAR $^{\circledR}$ (Takara Bio, Inc.) from human genomic DNA, and the mutant TGFBR2 3 'UTR was generated by Mut Express II Fast Mutagenesis kit V2 (VazymeBiotech Co., Ltd.). These DNA fragments were cloned into a psiCHECK $^{\mathrm{TM}}-2$ Vector (Promega Coproration). Plasmids were cut and joined by endonuclease QuickCut ${ }^{\mathrm{TM}}$ NotI, XhoI, and T4 DNA Ligase (Takara Bio, Inc.). $1 \times 10^{5}$ MDA-MB-231 cells were seeded in a 24-well plate and transfected, respectively, with the reporter constructs and miR-7 mimic (forward, 5'-UGGAAGACU AGUGAUUUUGUUGUU-3'; reverse, 5'-AACAACAAAAUC ACUAGUCUUCCA-3'), miR-7 mimic control (forward, 5'-UU UGUACUACACAAAAGUACUG-3'; reverse, 5'-CAGUAC UUUUGUGUAGUACAAA-3'), miR-7 inhibitor (5'-AAC 
A

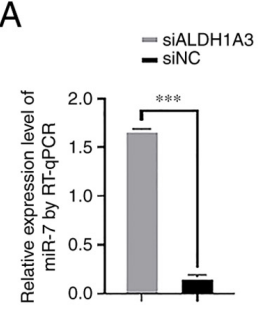

B

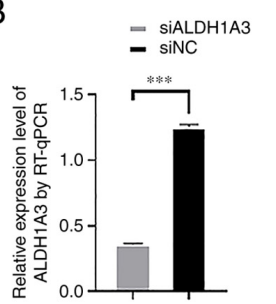

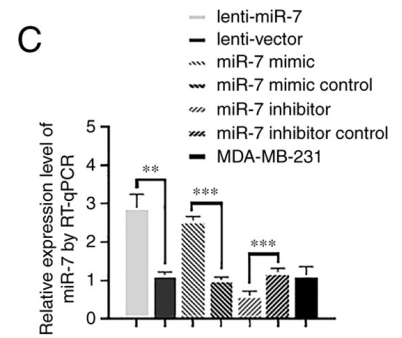
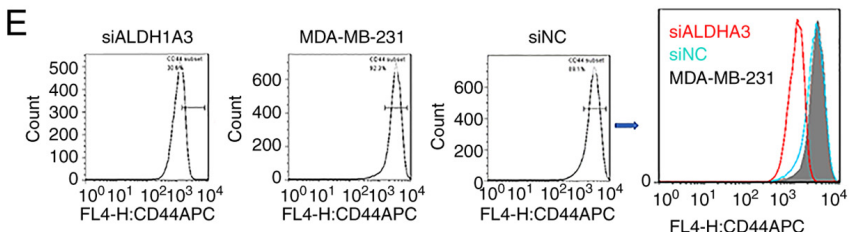

FL4-H:CD44APC

G


D
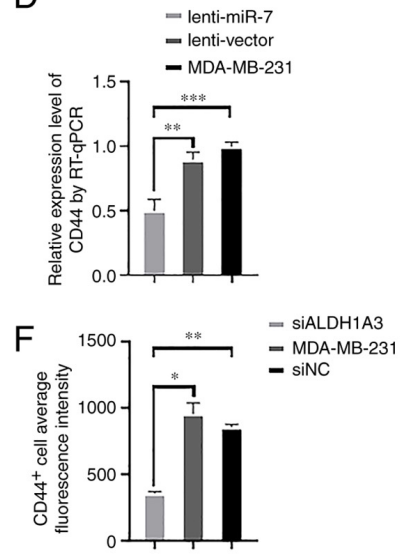

$\mathrm{H}$

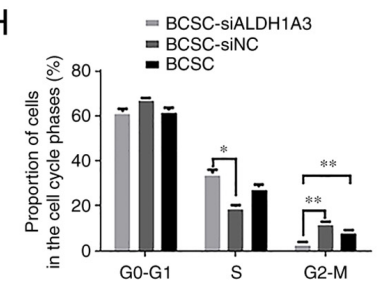

Figure 1. ALDH1A3 knockdown reduces CD44 expression in MDA-MB-231 cells and their proliferation in BCSCs. (A) Detection of miR-7 expression after ALDH1A3 knockdown in MDA-MB-231 cells by RT-qPCR. (B) Analysis of the effectiveness of ALDH1A3 knockdown in MDA-MB-231 cells. (C) Demonstration of transfection efficiency with lenti-miR-7, miR-7 mimic and miR-7 inhibitor. (D) Overexpression of miR-7 decreased CD44 gene expression. (E) siALDH1A3 reduced the ratio of $\mathrm{CD}_{4} 4^{+}$cells analyzed by FCM. (F) Statistical analysis revealed that the CD44+ cell average fluorescence intensity was reduced. (G) The proliferation of BCSC-siALDH1A3 cells was evaluated with FCM assay. (H) Statistical analysis of the cell cycle of BCSCs. "P $<0.05$, ${ }^{* *} \mathrm{P}<0.01$ and ${ }^{* * * *} \mathrm{P}<0.001$. ALDH1A3, aldehyde dehydrogenase 1 family member A3; BCSCs, breast cancer stem cells; RT-qPCR, reverse transcription-quantitative polymerase chain reaction; miR-7, microRNA-7; si, small interfering; FCM, flow cytometry.

AACAAAAUCACUAGUCUUCCA-3') and miR-7 inhibitor control (5'-CAGUACUUUUGUGUAGUACAAA-3'; all from Guangzhou RiboBio Co., Ltd.) for 48 h. Then, the luciferase reporter assay was performed using a Dual-Luciferase Reporter System (Promega Corporation).

Western blotting. Approximately $1 \times 10^{6}$ cells were harvested and lysed in RIPA lysis buffer (cat. no. P0013B; Beyotime Institute of Biotechnology), and the lysates were run on a western blot as previously described (12). The antibodies used for western blotting included CD44 (1:2,000 dilution; cat. no. 60224-1-Ig), Smad3 (1:2,000 dilution; cat. no. 66516-1-Ig), GAPDH (1:10,000 dilution; cat no. 60004-1-Ig) and TGFBR2 (1:2,000 dilution; cat. no. 66636-1-Ig) from ProteinTech Group, Inc. IRDye ${ }^{\circledR}$ 680RD donkey anti-mouse IgG secondary antibody (1:10,000 dilution; cat. no. P/N 926-68072) was from LI-COR Biosciences.

ChIP-PCR assays. The JASPAR software for bioinformatics prediction of transcription factors was used (http://jaspar. genereg.net/). The ChIP assay was performed according to the Chromatin Immunoprecipitation Kit Instruction Manual (EZ-ChIP ${ }^{\mathrm{TM}}$ cat. no. 17-371; Merck Millipore; Merck KGaA). The anti-Smad $2+$ Smad3 antibody (product code ab207447) was used to precipitate the protein-DNA complexes (Abcam) (13), and the DNA isolated through ChIP reactions was subjected to PCR using primers specific to the promoter of CD44 (forward, 5'-CCCAGATGGAGAAAGCTCTG-3'; reverse, 5'-ACTTGGCTTTCTGTCCTCCA-3').

Expression of lentivirus-infected cells and siRNA silencing gene. The three-plasmid system consisted of pSPAX2 $(10 \mu \mathrm{g})$, pMD2G $(5 \mu \mathrm{g})$, and pHBLV-U6-ZsGreen (15 $\mu \mathrm{g})$. miR-7 fragment (245 bp) was inserted into the lentivirus vector and cultured 48-72 $\mathrm{h}$ in 2nd generation 293T cells (Shanghai Institute of Biochemistry and Cell Biology), and the virus solution was obtained (plaque-forming units $/ \mathrm{ml}=0.033$; $\mathrm{MOI}=1$ ). MDA-MB-231 cells $\left(1 \times 10^{4}\right)$ were infected for $4 \mathrm{~h}$ with $10 \mu \mathrm{l}$ lenti-miR-7 virus solution and miR-7 overexpression monoclonal cells were selected after 5 days. siRNA and miR-7 mimic were synthesized by the Guangzhou RiboBio Co., Ltd. and used as previously described (8). A total of $50 \mathrm{nM}$ siRNA/miR-7 mimic/inhibitor and negative controls were used to transfect cells.

Statistical analysis. SPSS Statistics 21.0 (IBM Corp.) and GraphPad Prism 8.0 (GraphPad Software, Inc.) were used for data analysis and imaging. Values of interest were presented as the mean \pm standard deviation. Statistical analyses were performed using Tukey's post hoc test after one-way analysis of variance (ANOVA) for multiple comparisons, and Spearman's correlation analysis. Values shown are from one representative experiment. $\mathrm{P}<0.05$ was considered to indicate a statistically significant difference. 

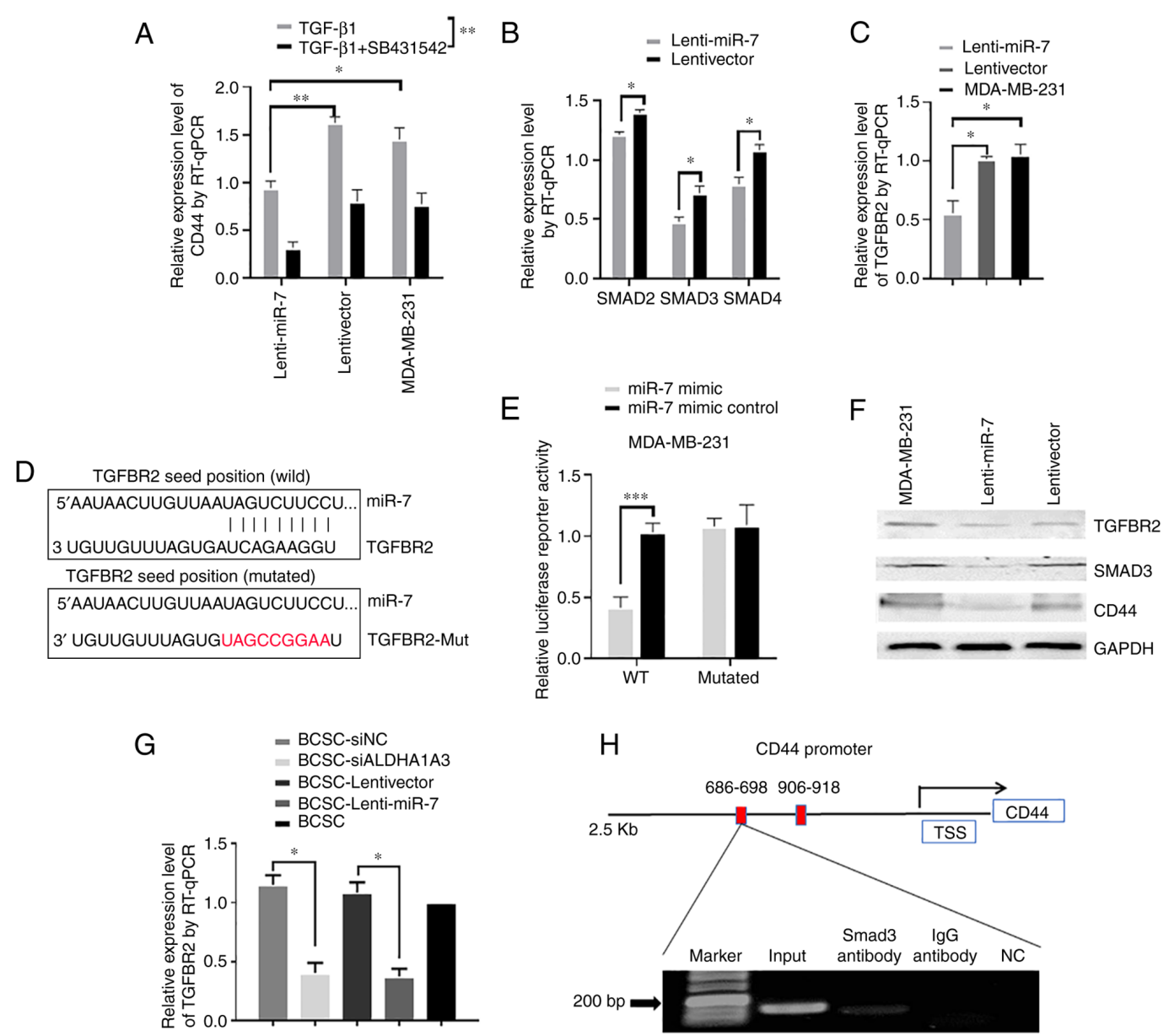

Figure 2. miR-7 directly targets TGFBR2 and Smad3 binds to the CD44 promoter region. (A) Lenti-miR-7 and lentivector MDA-MB-231 cells were respectively incubated with TGF- $\beta 1$ and TGF- $\beta 1+$ SB431542, and then CD44 mRNA relative expression levels were measured by RT-qPCR. (B) The relative expression levels of Smad2, Smad3, and Smad4 were measured by RT-qPCR in lenti-miR-7 and lentivector MDA-MB-231 cells. (C) TGFBR2 mRNA relative expression levels were measured by RT-qPCR. (D) TGFBR2 3'UTR with the miR-7 binding site was predicted, and complementary sequences of miR-7 to TGFBR2 3'UTR mutated are presented in red. (E) Cells were harvested and luciferase activities were measured after 48-h transfection. (F) The expression levels of TGFBR2, Smad3, and CD44 were analyzed by western blotting. (G) TGFBR2 mRNA relative expression levels were measured by RT-qPCR in BCSC-siALDH1 A3, BCSC-lenti-miR-7, and control cells. (H) ChIP-PCR assay in MDA-MB-231 cells. ${ }^{*} \mathrm{P}<0.05,{ }^{* *} \mathrm{P}<0.01$ and ${ }^{* * * *} \mathrm{P}<0.001$. miR-7, microRNA-7; TGFBR2, transforming growth factor- $\beta$ receptor 2 ; RT-qPCR, reverse transcription-quantitative polymerase chain reaction; BCSC, breast cancer stem cell; si, small interfering.

\section{Results}

Inhibition of ALDH1A3 decreases CD44 expression. miR-7 and ALDH1A3 expression was detected by RT-qPCR after knocking down ALDH1A3 with siRNA, and it was revealed that miR-7 expression was significantly increased (Fig. 1A) and ALDH1A3 expression was significantly decreased (Fig. 1B). The results confirmed that not only could miR-7 regulate ALDH1A3 (9) but ALDH1A3 could also reversely regulate miR-7 expression. To demonstrate transfection efficiency, lenti-miR-7 and miR-7 mimic were transfected into MDA-MB-231 cells, and RT-qPCR results revealed that they could significantly increase the expression of miR-7, while the miR-7 inhibitor could inhibit the expression of miR-7 (Fig. 1C). Using a lentivirus to overexpress miR-7 (lenti-miR-7) could significantly reduce the expression of CD44 mRNA (Fig. 1D). Then, the ratio of $\mathrm{CD}_{4} 4^{+}$cells in the MDA-MB-231 cells was examined by FCM after knocking down ALDH1A3 with siRNA (Fig. 1E). As revealed in Fig. 1F, the ratio of $\mathrm{CD} 44^{+}$cells was significantly decreased compared to the control group. In order to further evaluate the effect of siALDH1A3 on cell proliferation, FCM was used to analyze the cell cycle of BCSCs (Fig. 1G). Cell cycle analysis revealed that BCSC-siALDH1A3 increased the S phase and reduced the $\mathrm{G} 2 / \mathrm{M}$ phase compared to the BCSC-siNC cell population (Fig. 1H). Collectively, the knockdown of ALDH1A3 expression reduced the CD44 expression in MDA-MB-231 cells and their in vitro proliferation in BCSCs.

miR-7 overexpression inhibits TGF- $\beta 1$ signaling pathway and downregulates $C D 44$ expression. To demonstrate whether miR-7 inhibits the TGF- $\beta 1$ signaling pathway, lenti-miR-7 and lentivector cells were treated with $10 \mathrm{ng} / \mathrm{ml}$ TGF- $\beta 1$ and $100 \mathrm{ng} / \mathrm{ml}$ TGF- $\beta 1$ type I receptor antagonist SB431542. RT-qPCR results revealed that miR-7 inhibited the effect of TGF- $\beta 1$-upregulation of CD44. However, SB431542 could enhance the effect of miR-7 concomitantly by inhibiting TGF- $\beta 1$, compared with the control group (Fig. 2A). Next, RT-qPCR was used to evaluate the effect of miR-7 on the main signaling molecules of the TGF- $\beta 1$ signaling pathway. 
A

- Adjacent normal ]*


$\mathrm{B}$



D

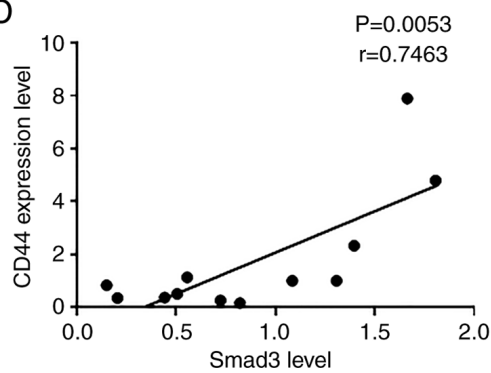

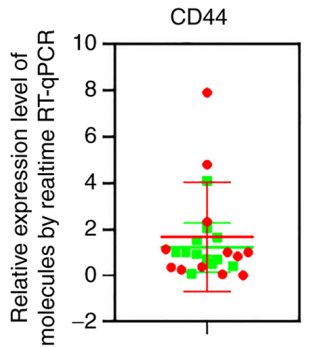

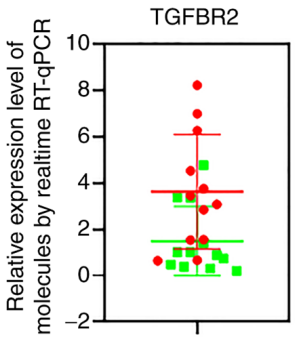

C

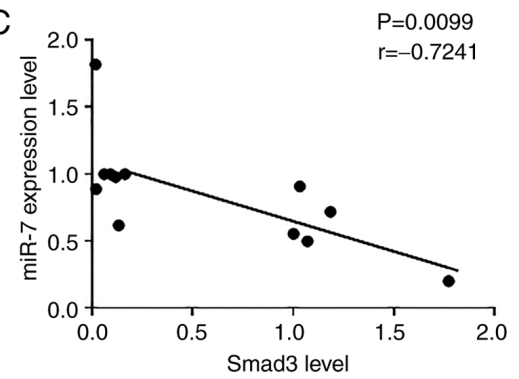

E

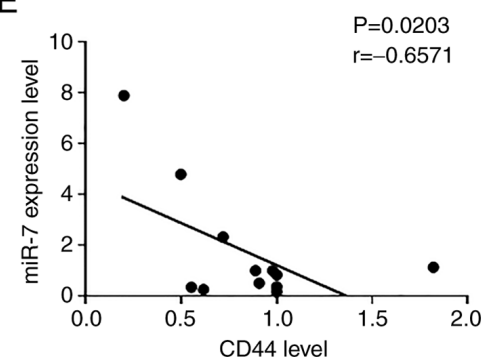

Figure 3. Detection of miR-7 and BCSC-related molecular expression in breast cancer surgical specimens. (A) Relative expression levels of miR-7, Smad3, CD44, and TGFBR2 in breast cancer postsurgery samples analyzed by RT-qPCR. (B-E) Relative expression levels of miR-7 and TGFBR2, miR-7 and Smad3, Smad3 and CD44, and miR-7 and CD44, respectively. The green points represent adjacent noncancerous tissues; the red points represent tumor tissues ( $\mathrm{n}=12$ ). "P<0.05. miR-7, microRNA-7; BCSC, breast cancer stem cell; TGFBR2, transforming growth factor- $\beta$ receptor 2; RT-qPCR, reverse transcription-quantitative polymerase chain reaction.

As revealed in Fig. 2B, miR-7 downregulated the expression levels of Smad2, Smad3, and Smad4. In addition, as indicated in Fig. 2C, miR-7 also downregulated the expression of TGFBR2. To demonstrate the targeted regulatory relationship between miR-7 and TGFBR2, the targeted binding sites of miR-7 and TGFBR2 3'UTR were predicted through the bioinformatics website (Fig. 2D). For this reason, the MDA-MB-231 cells were transfected with psiCHECK-2-TGFBR2 and psiCHECK-2-TGFBR2-Mut dual-luciferase recombinant plasmids using Lipofectamine 2000, respectively. As revealed in Fig. 2E, the luciferase activity of the dual-luciferase recombinant plasmid was not altered in the TGFBR2 3'UTR mutation group, while the luciferase activity of the wild-type dual-luciferase recombinant plasmid exhibited a significant decrease, indicating that miR-7 mimic could effectively bind to TGFBR2 3'UTR and reduce the relative luciferase activity of the wild-type vector. Western blot results further demonstrated that miR-7 downregulated TGFBR2, Smad3, and CD44 (Fig. 2F). Furthermore, siALDH1 A3 and lenti-miR-7 were transfected with Lipofectamine 2000 separately to verify the expression of TGFBR 2 in the differently treated
BCSCs. Compared to the control (BCSC-lentivector), the expression of TGFBR2 in BCSC-lenti-miR-7 group was decreased (Fig. 2G). Finally, the ChIP-PCR assay was performed. Bioinformatics predicted that the transcription factor Smad3 binds to the upstream region of the CD44 gene promoter $(14,15)$. Immunoprecipitation was performed with the Smad3 antibody, and DNA fragments were eluted from the immunoprecipitation complex. The identification of a positive amplification product indicated that $\mathrm{Smad} 3$, a transcription factor, could regulate gene expression by binding the upstream region of the CD44 promoter (Fig. 2H).

Demonstration of miR-7-TGFBR2-Smad3-CD44 axis. It was further evaluated whether there is a miR-7-TGFBR2Smad3-CD44 axis in both breast cancer cell lines and breast cancer surgical specimens. First, surgical tissues were obtained from 12 breast cancer patients and RT-qPCR was used to detect the expression levels of miR-7, Smad3, CD44 and TGFBR2. RT-qPCR results revealed that the relative expression level of miR-7 was expressed at a lower level in breast cancer tissues compared with adjacent noncancerous 
A


B

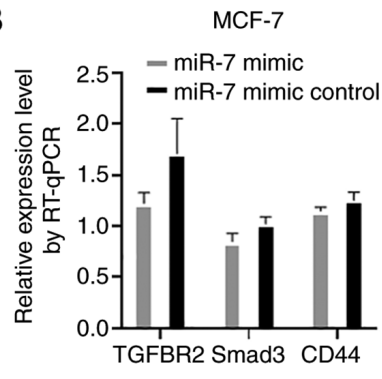

$E$

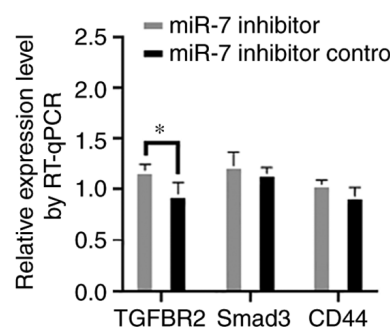

C

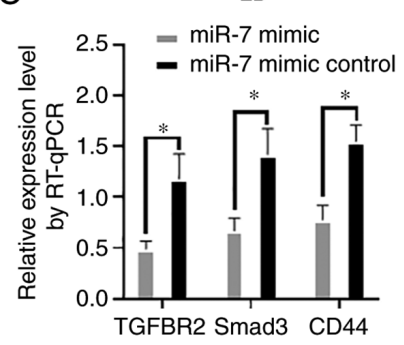

$\mathrm{F}$

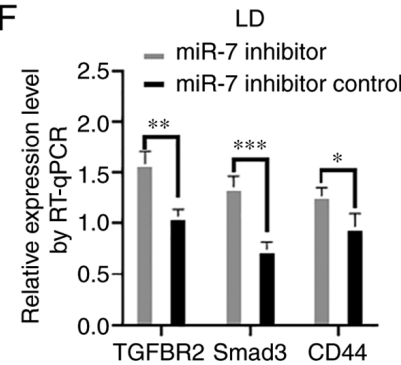

Figure 4. Detection of the molecular expression in SK-BR-3, MCF-7, and LD breast cancer cell lines. (A-C) TGFBR2, Smad3, and CD44 expression levels in SK-BR-3, MCF-7, and LD cells were measured by RT-qPCR analysis after miR-7 mimic transfection. (D-F) TGFBR2, Smad3, and CD44 relative expression levels in SK-BR-3, MCF-7, and LD cells were detected after the inhibitor transfection by RT-qPCR. ${ }^{*} \mathrm{P}<0.05,{ }^{* *} \mathrm{P}<0.01$ and ${ }^{* * * *} \mathrm{P}<0.001$. TGFBR2, transforming growth factor- $\beta$ receptor 2 ; RT-qPCR, reverse transcription-quantitative polymerase chainreaction; miR-7, microRNA-7.

tissues, while the relative expression levels of TGFBR2, CD44, and Smad3 were significantly higher in breast cancer tissues than in adjacent noncancerous tissues, as revealed in Fig. 3A. It was determined that miR-7 was negatively correlated with TGFBR2 (Fig. 3B), CD44 (Fig. 3E), and Smad3 (Fig. 3C). Smad3 was positively correlated with CD44 (Fig. 3D). Second, the relative expression levels of TGFBR2, Smad3, and CD44 were concurrently analyzed in breast cancer cell lines SK-BR-3, MCF-7, and LD using RT-qPCR after miR-7 mimic transfection. As revealed in Fig. 4A-C, the relative expression levels of TGFBR2, Smad3, and CD44 were all downregulated in the miR-7-mimic-transfected cells. In contrast, the mRNA expression levels of TGFBR2, Smad3, and CD44 were all upregulated after miR-7 inhibitor transfection (Fig. 4D-F). These results suggested that the miR-7-TGFBR2-Smad3-CD44 axis exists objectively in both breast cancer cell lines and breast cancer surgical specimens.

\section{Discussion}

The previous results of our research group revealed that the overexpression of miR-7 caused the downregulation of ALDH1A3 and CD44 (9). However, how miR-7 downregulates CD44 and what role ALDH1A3 plays in impacting CD44 expression was not elucidated. Therefore, the molecular mechanism that affects the expression of CD44 was explored by regulating ALDH1A3 in the present study.

ALDH1A3 is the major isozyme that contributes to ALDH enzyme activities in 58 human cancer cell lines. In breast cancer, glioma, and melanoma, ALDH1A3 expression is regulated by several mechanisms at epigenetic, transcriptional, and posttranslational levels (16-18). Attenuation of ALDH1A3 expression by RNA interference (RNAi) significantly suppressed cell proliferation, reduced the number of cancer cells that persisted after anticancer drug treatment, and interfered with tumor growth in a mouse xenograft model (17). Our previous study (9) revealed that miR-7 expression could downregulate ALDH1A3 expression. RT-qPCR experiments revealed that miR-7 expression was upregulated by downregulating ALDH1A3 expression using siRNA. These experimental results suggested that there is a mutual regulation between miR-7 and ALDH1A3. To further understand and reveal the relationship between miR-7 and ALDH1A3, further investigation was carried out.

The cell surface protein CD44 has been widely used as a CSC marker in breast cancer and various other types of cancers $(19,20)$. CD44 is important for tumor initiation in vivo and predominantly expressed in metastatic breast cancer cells. Previous research results have also revealed that in these metastatic breast cancer cell lines, knockdown of CD44 significantly inhibited breast cancer metastasis $(21,22)$. Therefore, it is of great significance to explore the mechanism of miR-7 inhibition of CD44 and reduce the tumorigenicity of BCSCs.

To better understand the relationship between miR-7 and CD44, a bioinformatics approach was used to predict whether miR-7 regulates CD44 cell surface expression via the TGF- $\beta 1$ signaling pathway. Therefore, confirmation that the TGF- $\beta 1$ signaling pathway is regulated by miR-7 and affects CD44 gene expression was first required. TGF- $\beta 1$ $(10 \mathrm{ng} / \mathrm{ml})$ and TGF- $\beta 1$ type I receptor antagonist SB431542 $(100 \mathrm{ng} / \mathrm{ml})$ were used to treat lenti-miR-7 and lentivector cells by Lipofectamine 2000, respectively (23). After $72 \mathrm{~h}$, it was revealed that TGF- $\beta 1$ could significantly increase the intracellular CD44 mRNA expression. On this basis, the inhibition of TGF- $\beta 1$ by SB 431542 could significantly reduce CD 44 mRNA expression in MDA-MB-231 cells. Compared with lentivector cells, the expression of CD44 mRNA was lower in the case of miR-7 overexpression in lenti-miR-7 cells. The aforementioned results revealed that the TGF- $\beta 1$ signaling pathway was involved in the regulation of CD44. Next, the expression levels 
of Smad2, Smad3, and Smad4 in lenti-miR-7 cells were examined. It was revealed that, in addition to the inhibitory factor, Smad2, Smad3, and Smad4 all exhibited decreased mRNA expression. This further suggested that TGF- $\beta 1$ signaling was regulated by miR-7 overexpression.

To explain how miR-7 inhibits the TGF- $\beta 1$ signaling pathway, bioinformatics were used to predict whether miR-7 has binding targets for TGFBR2 3'UTR. It is well known that TGF- $\beta$ ligands assemble their corresponding receptors that contain two type 1 components and two type 2 components. Type 2 receptors serve as activators to phosphorylate type I receptors, whereas type 1 receptors function as propagators to transduce the downstream signal to cytoplasmic proteins (24). The components of both receptors are serine/threonine kinases. TGF- $\beta$ type I receptors and activin type 1 receptors phosphorylate SMAD2/3 (25). The expression of TGFBR2 by RT-qPCR was first detected and it was revealed that, in the case of miR-7 overexpression, TGFBR 2 mRNA expression was downregulated. The results of RT-qPCR were further confirmed by western blotting, suggesting miR-7 affects TGFBR2 expression.

The psiCHECK-2-TGFBR2 and psiCHECK-2-TGFBR2Mut dual-luciferase reporters were constructed, respectively. The psiCHECK ${ }^{\mathrm{TM}}-2$ vector was designed by Promega Coproration to provide a quantitative and rapid approach for the optimization of RNAi. The vectors enable the monitoring of changes in the expression of a target gene fused to the reporter gene, containing as the primary reporter gene the synthetic version of Renilla luciferase, hRluc (26). This synthetic gene is engineered for more efficient expression in mammalian cells and for reduced anomalous transcription (27). After transfecting miR-7 mimic and dual-luciferase reporters into MDA-MB-231 cells, the luciferase activity of wild-type cells significantly decreased, while the luciferase activity of mutant cells was not significantly altered, indicating that miR-7 can bind to TGFBR2 3'UTR. Concurrently, the mRNA expression of TGFBR2 in BCSCs that were isolated from MDA-MB-231 cells according to the phenotypes of $\mathrm{CD}^{4} 4^{+} \mathrm{CD} 24{ }^{-} \mathrm{ESA}^{+} \mathrm{BCSC}$ was also observed (26). The cDNA products of these BCSCs had also been used in a previously published study (9). The results in the present study revealed that the mRNA expression of TGFBR2 in BCSC in the siALDH1A3 and lenti-miR-7 groups was visibly lower than that in the control group.

Genome-wide identification of transcription factor binding sites (TFBSs) is key to understanding transcriptional regulation. The genomic locations where transcription factors bind to DNA are typically short $(6-20 \mathrm{bp})$ and exhibit sequence variability (28). The DNA sequence of the 2,500-bp region upstream of the CD44 promoter was obtained, Smad3 was used as a transcription factor for motif binding prediction, and the TFBSs for research based on the motif conservation score were selected (14). The ChIP-PCR analysis strongly revealed that the Smad3 protein binds to the 686-698 position upstream of the CD44 promoter. The results demonstrated that miR-7 affects the TGF- $\beta 1$ signaling pathway molecule Smad3 by downregulating TGFBR2 and then inhibits CD44 gene transcription. Collectively, the present study identified miR-7-TGFBR2-Smad3-CD44 as a regulatory axis of BCSC marker CD44 expression.
RT-qPCR experiments were performed on cancer tissues and adjacent tissues from 12 clinical breast cancer surgical samples. It was revealed that, compared with adjacent tissues, miR-7 in cancer tissues exhibited low expression, and TGFBR2, Smad3, and CD44 exhibited high expression. These molecular relationships were analyzed, and it was determined that miR-7 was negatively correlated with TGFBR2, Smad3 and CD44 respectively, and that CD44 and Smad3 were positively correlated. Then, SK-BR-3, MCF-7, and LD breast cancer cell lines were used to analyze the effects of miR-7 on the miR-7-TGFBR2-Smad3-CD44 axis. In the case of miR-7 overexpression, TGFBR2, Smad3, and CD44 were all downregulated; while by inhibiting miR-7 expression, all three molecules, TGFBR2, Smad3, and CD44 were upregulated in the breast cancer cell lines.

Collectively, the present findings revealed that the downregulation of ALDH1A3 can upregulate miR-7 and reduce the ratio of $\mathrm{CD} 44^{+}$cells in breast cancer cells via the miR-7-TGFBR2-Smad3-CD44 axis. Notably, these findings have potential clinical importance for understanding the multiple regulatory roles of miR-7. Inhibiting ALDH1A3 and/or miR-7 overexpression may be an important method for treating breast cancer.

\section{Acknowledgements}

Not applicable.

\section{Funding}

The present study was supported by the National Natural Science Foundation of China (grant no. 81572887), and partly supported by the National Key Research and Development Program of China (grant no. 2017YFA0205502), the Scientific Research Foundation of Graduate School of Southeast University (grant no. YBJJ1849), and the Postgraduate Research \& Practice Innovation Program of Jiangsu Province (grant no. KYCX18_0165).

\section{Availability of data and materials}

The datasets used and/or analyzed during the current study are available from the corresponding author on reasonable request.

\section{Authors' contributions}

JD designed the experiments and MP, ML, MG, HZ carried out the experiments, data analysis and manuscript writing. HX, FZ, FM and RX performed reverse transcription PCR, western blotting and flow cytometry assays. MP and ML performed ChIP-PCR and dual-luciferase assays. JD interpreted the data, edited and corrected the manuscript and approved the final version to be published. JD and MP confirmed the authenticity of all the raw data. All authors read and approved the final manuscript.

\section{Ethics approval and consent to participate}

The investigation was approved by the Ethics Committee at Southeast University School of Medicine (Nanjing, China), 
and informed consent for the use of the postsurgery samples was obtained from the donors who were breast cancer patients.

\section{Patient consent for publication}

Not applicable.

\section{Competing interests}

The authors declare that they have no competing interests.

\section{References}

1. Gonzalez-Angulo AM, Morales-Vasquez F and Hortobagyi GN: Overview of resistance to systemic therapy in patients with breast cancer. Adv Exp Med Biol 608: 1-22, 2007.

2. Chaffer CL and Weinberg RA: A perspective on cancer cell metastasis. Science 331: 1559-1564, 2011.

3. Dou J, Li Y, Zhao F, Hu W, Wen P, Tang Q, Chu L, Wang Y, Cao M, Jiang $\mathrm{C}$ and $\mathrm{Gu} \mathrm{N}$ : Identification of tumor stem-like cells in a mouse myeloma cell line. Cell Mol Biol (Noisy-le-grand) 55 (Suppl): OL1151-OL1160, 2009.

4. Bravo-Egana V, Rosero S, Molano RD, Pileggi A, Ricordi C, Domínguez-Bendala J and Pastori RL: Quantitative differential expression analysis reveals miR-7 as major islet microRNA. Biochem Biophys Res Commun 366: 922-926, 2008.

5. Junn E, Lee KW, Jeong BS, Chan TW, Im JY and Mouradian MM: Repression of alpha-synuclein expression and toxicity by microRNA-7. Proc Natl Acad Sci USA 106: 13052-13057, 2009.

6. Li X and Carthew RW: A microRNA mediates EGF receptor signaling and promotes photoreceptor differentiation in the Drosophila eye. Cell 123: 1267-1277, 2005.

7. Zhang H, Cai K, Wang J, Wang X, Cheng K, Shi F, Jiang L, Zhang Y and Dou J: miR-7, inhibited indirectly by lincRNA HOTAIR, directly inhibits SETDB1 and reverses the EMT of breast cancer stem cells by downregulating the STAT3 pathway. Stem Cells 32: 2858-2868, 2014.

8. Li M, Pan M, You C, Zhao F, Wu D, Guo M, Xu H, Shi F, Zheng D and Dou J: miR-7 reduces the BCSC subset by inhibiting XIST to modulate the miR-92b/Slug/ESA axis and inhibit tumor growth. Breast Cancer Res 22: 26, 2020.

9. Pan M, Li M, You C, Zhao F, Guo M, Xu H, Li L, Wang L and Dou J: Inhibition of breast cancer growth via miR-7 suppressing ALDH1A3 activity concomitant with decreasing breast cancer stem cell subpopulation. J Cell Physiol 235: 1405-1416, 2020.

10. Livak KJ and Schmittgen TD: Analysis of relative gene expression data using real-time quantitative PCR and the 2(-Delta Delta C(T)) method. Methods 25: 402-408, 2001.

11. Mon-López D and Tejero-González CM: Validity and reliability of the TargetScan ISSF Pistol \& Rifle application for measuring shooting performance. Scand J Med Sci Sports 29: 1707-1712, 2019

12. Dou J, Wang Y, Yu F, Yang H, Wang J, He X, Xu W, Chen J and Hu K: Protection against Mycobacterium tuberculosis challenge in mice by DNA vaccine Ag85A-ESAT-6-IL-21 priming and BCG boosting. Int J Immunogenet 39: 183-190, 2012.

13. Cibi DM, Mia MM, Guna Shekeran S, Yun LS, Sandireddy R, Gupta P, Hota M, Sun L, Ghosh S and Singh MK: Neural crest-specific deletion of Rbfox 2 in mice leads to craniofacial abnormalities including cleft palate. Elife 8: e45418, 2019.

14. Fornes O, Castro-Mondragon JA, Khan A, van der Lee R, Zhang X, Richmond PA, Modi BP, Correard S, Gheorghe M, Baranašić D, et al: JASPAR 2020: Update of the open-access database of transcription factor binding profiles. Nucleic Acids Res 48 (D1): D87-D92, 2020.
15. KhanA,Fornes O,Stigliani A,GheorgheM,Castro-MondragonJA, van der Lee R, Bessy A, Chèneby J, Kulkarni SR, Tan G, et al: JASPAR 2018: Update of the open-access database of transcription factor binding profiles and its web framework. Nucleic Acids Res 46 (D1): D260-D266, 2018.

16. Kawakami R, Mashima T, Kawata N, Kumagai K, Migita T, Sano T, Mizunuma N, Yamaguchi $\mathrm{K}$ and Seimiya $\mathrm{H}$ : ALDH1A3-mTOR axis as a therapeutic target for anticancer drug-tolerant persister cells in gastric cancer. Cancer Sci 111: 962-973, 2020.

17. Yamashita D, Minata M, Ibrahim AN, Yamaguchi S, Coviello V, Bernstock JD, Harada S, Cerione RA, Tannous BA, La Motta C, et al: Identification of ALDH1A3 as a viable therapeutic target in breast cancer metastasis-initiating cells. Mol Cancer Ther 19: 1134-1147, 2020.

18. Wang S, Zhou X, Liang C, Bao M, Tian Y, Zhu J, Zhang T, Yang J and Wang Z: ALDH1A3 serves as a predictor for castration resistance in prostate cancer patients. BMC Cancer 20: 387, 2020.

19. Al-Hajj M, Wicha MS, Benito-Hernandez A, Morrison SJ and Clarke MF: Prospective identification of tumorigenic breast cancer cells. Proc Natl Acad Sci USA 100: 3983-3988, 2003. Erratum in: Proc Natl Acad Sci USA 100: 6890, 2003.

20. Fillmore $C$ and Kuperwasser C: Human breast cancer stem cell markers CD44 and CD24: Enriching for cells with functional properties in mice or in man? Breast Cancer Res 9: 303, 2007.

21. Zhao P, Xu Y, Wei Y, Qiu Q, Chew TL, Kang Y and Cheng C: The CD44s splice isoform is a central mediator for invadopodia activity. J Cell Sci 129: 1355-1365, 2016.

22. Zhang H, Brown RL, Wei Y, Zhao P, Liu S, Liu X, Deng Y, $\mathrm{Hu} \mathrm{X}$, Zhang J, Gao XD, et al: CD44 splice isoform switching determines breast cancer stem cell state. Genes Dev 33: 166-179, 2019.

23. Sun C, Sun L, Jiang K, Gao DM, Kang XN, Wang C, Zhang S, Huang S, Qin X, Li Y, et al: NANOG promotes liver cancer cell invasion by inducing epithelial-mesenchymal transition through NODAL/SMAD3 signaling pathway. Int J Biochem Cell Biol 45: 1099-1108, 2013. Erratum in: Int J Biochem Cell Biol 105: 144 2018.

24. Itatani Y, Kawada K and Sakai Y: Transforming growth factor- $\beta$ signaling pathway in colorectal cancer and its tumor microenvironment. Int J Mol Sci 20: E5822, 2019.

25. Vitiello GAF, Amarante MK, Banin-Hirata BK, Campos CZ, de Oliveira KB, Losi-Guembarovski R and Watanabe MAE: Transforming growth factor beta receptor II (TGFBR2) promoter region polymorphism in Brazilian breast cancer patients: Association with susceptibility, clinicopathological features, and interaction with TGFB1 haplotypes. Breast Cancer Res Treat 178: 207-219, 2019.

26. Kumar R, Conklin DS and Mittal V: High-throughput selection of effective RNAi probes for gene silencing. Genome Res 13: 2333-2340, 2003.

27. Zhao L, Zhang Y and Zhang Y: Long noncoding RNA CASC2 regulates hepatocellular carcinoma cell oncogenesis through miR-362-5p/Nf-kB axis. J Cell Physiol 233: 6661-6670, 2018.

28. Xie X, Lu J, Kulbokas EJ, Golub TR, Mootha V, Lindblad-Toh K, Lander ES and Kellis M: Systematic discovery of regulatory motifs in human promoters and 3' UTRs by comparison of several mammals. Nature 434: 338-345, 2005.



This work is licensed under a Creative Commons Attribution-NonCommercial-NoDerivatives 4.0 International (CC BY-NC-ND 4.0) License. 